Article

\title{
Distributed Event-Based Control of Hierarchical Leader-Follower Networks with Time-Varying Layer-To-Layer Delays
}

\author{
Guang-Hui Xu ${ }^{1,2}$, Meng Xu ${ }^{1,2}$, Ming-Feng Ge ${ }^{3, *}$, Teng-Fei Ding ${ }^{3}$, Feng Qi ${ }^{1,2}$ and Meng Li ${ }^{1,2}$ \\ 1 School of Electrical and Electronics Engineering, Hubei University of Technology, Wuhan 430068, China; \\ xgh@hbut.edu.cn (G.-H.X.); 101710208@hbut.edu.cn (M.X.); 101810271@hbut.edu.cn (F.Q.); \\ 101810207@hbut.edu.cn (M.L.) \\ 2 Hubei Key Laboratory for High-Efficiency Utilization of Solar Energy and Operation Control of Energy \\ Storage System, Hubei University of Technology, Wuhan 430068, China \\ 3 School of Mechanical Engineering and Electronic Information, China University of Geosciences, \\ Wuhan 430074, China; dingtf@cug.edu.cn \\ * Correspondence: gemf@cug.edu.cn or fmgabc@163.com
}

Received: 12 February 2020; Accepted: 3 April 2020; Published: 9 April 2020

\begin{abstract}
Compared with the traditional multi-agent models, the hierarchical leader-follower network (HLFN) can describe some real-world multi-agent systems more precisely due to its layered properties. The distributed event-based consensus control problem of HLFNs with layer-to-layer delays, namely, communication delays among agents of different layers, is presented in this essay. In order to solve the aforementioned problem, several innovative hierarchical event-based control (HEC) algorithms are proposed. The sufficient conditions on the control parameters and event-triggered mechanism were derived to undertake the reliability of the closed-loop dynamics. Moreover, it is shown that the zeno-behaviors of the presented HEC algorithms can be excluded. Finally, there are some numerical examples that verify the availability of the results.
\end{abstract}

Keywords: event-based consensus; hierarchical leader-follower network; hierarchical event-based control; layer-to-layer delays

\section{Introduction}

In recent years, coordination problems of multi-agent systems (MAS) have attracted research interests, which cover a wide area such as flocking [1,2], formation control [3-5], consensus tracking of a robot [6], and rendezvous in distributed robotics [7-9]. On these issues, many remarkable and significant results have emerged one after another.

In recent years, the research on the consensus of the leader-following multi-agent system has been increasing, and a large number of achievements have emerged. Wang et al. [10] investigated the leader-following consensus by a self-triggered algorithm. At the same time, considering the existence of a disturbance in reality, the input delay was considered. Shen et al. [11] investigated the leader-following consensus problem of multi-agent systems with two distributed adaptive control schemes. Ning [12] investigated the problem of leader-following consensus for multiple wheeled mobile robots. Based on the above research,the multi-layer hierarchical structure on the basis of the two-layer structure was investigated. The hierarchical structure exists widely in nature. In reality, birds show a hierarchical structure in the process of migration, and each layer is both a leader layer and follow layer, in addition to the leader of the first layer and the last follow layer, this model is called a hierarchical leader-follower network (HLFN). Nagy [13] showed the hierarchical piloting behavior of a group of pigeons during a long flight for the first time. Through the way of recording the 
experimental data, it can be found that the pigeons have established a hierarchical leadership structure to interact with each other during the flight, so as to complete the whole long-distance flight mission effectively. He and $\mathrm{Xu}$ [14] solved the coordination problem of MAS with two-layer leaders. Based on the previous work, we investigated the consensus of HLFNs.

In the actual communication network, due to the limitations of distance, bandwidth, and sensors, time delays between nodes are inevitable. Based on the passivity theory, the state estimation of recurrent neural networks (MRNNs) with time-varying delays is studied in [15]. Li [16] investigated the leader-following consensus of MASs with time delays and switching topology. Zhao [17] studied the consensus problem for MASs with communication delays. Jiang [18] solved the consensus problem of networked multiple robotic manipulators with and without time-varying delays. However, in large hierarchical networks, the communication delays between layers (layer-to-layer delays) are often different, and the different types of delays between different layers have not been solved.

In practical applications, continuous communication will lead to high communication costs and high requirements for equipment. Therefore, the event-based control has received widespread attentions as a method which can effectively reduce the cost of communication. In event-based control, the controller update only depends on the designed trigger conditions, and the trigger conditions are often related to the state of the system. The bipartite consensus problem of multi-agent system with connected structure balanced symbol graph based on event trigger was studied in paper [19]. Yi [20] has investigated the global consensus problem for first-order continuous-time multi-agent systems with input saturation, in which the triggering law does not require any a priori knowledge of global network parameters. The paper [21] investigates the bipartite leader-following consensus of second-order multi-agent systems with signed digraph topology. The paper [22] showed that event trigger control has lower communication times and controller update times and better control effect than periodic sampling control on the premise of completing control tasks. The event-based control technology attracted much attention because of the characteristics of resource saving, and related research also achieved more results [23-30]. However, the consensus problem of HLFNs remains unsolved.

Inspired by the preceding discussions, it is desirable to design economical and efficient hierarchical event-based control (HEC) algorithms for consensus problems of HLFNs with layer-to-layer delays. The main contributions of our paper are twofold: (1) Compared with the existing research achievements [30-34], this essay deals with the consensus problem of HLFNs with layer-to-layer delays. The existing literature considers the time-delays between different agents at the same layer. In order to better study the hierarchical structure, we considered layer-to-layer delays between different layers. (2) Based on the particularity of hierarchical structure, a new HEC algorithm was proposed to solve the previous problem. The algorithm is different from the general event-based method, as the trigger conditions of each layer were different.

In the rest of this paper is structured as follows: Section 2 introduces several fundamental theories of graph and matrix, and the three-layer MAS is described. In Section 3, the convergence of the designed control law is analyzed in detail. A numerical example is given to present the effectiveness of designed event-triggered mechanism in Section 4. Section 5 concludes this paper.

\section{Preliminaries}

\subsection{Basic Theory on Graphs and Matrices}

A directed graph $\mathcal{G}=\{\mathcal{V}, \mathcal{E}, \mathcal{A}\}$ consists of a node set $\mathcal{V}=\left\{v_{1}, v_{2}, \cdots, v_{N}\right\}$, an edge set $\mathcal{E} \in$ $(\mathcal{V} \times \mathcal{V})$, and a weighted adjacency matrix $\mathcal{A}=\left(a_{i j}\right)$. If there is a edge between nodes $i$ and $j$, then $(i, j) \in \mathcal{E} \Leftrightarrow a_{i j}>0$. There are no self-loops, i.e., $a_{i i}=0$. The neighbor set of agent $i$ is defined as $\mathcal{N}_{i}=\{j \in \mathcal{V} \mid(j, i) \in \mathcal{E}, j \neq i\}$. Besides, the Laplacian matrix $\mathcal{L}$ is defined as $\mathcal{L}=\left[l_{i j}\right]$, where $l_{i i}=\sum_{j \neq i} a_{i j}$ and $l_{i j}=-a_{i j}, i \neq j$. Further, $D_{k}=\operatorname{diag}\left(d_{1}, d_{2}, \ldots, d_{r_{k}}\right), k=2,3, \ldots, l, r_{k}$ is the number of 
nodes of $k$ th layer. $1+r_{2}+\ldots+r_{l}=N$. If the node of $k$ th layer can receive information from the $r_{k-1}$, then $d_{r_{k}}>0$, otherwise $d_{r_{k}}=0$.

\subsection{System Description}

Without loss of generality, the HLFNs contain three-layer network in this paper. The first layer contains only one leader (as node 0 ), the second layer contains $r_{2}$ minor leader, the third layer contains $m$ groups, $r_{3}$ followers, and each group can receive information from the minor leader layer. Let the topological relationship between the minor leaders be $\mathcal{G}_{2}$, its corresponding Laplacian matrix is $\mathcal{L}_{2}$. Followers are divided into $m$ subgroups, and $\mathcal{G}_{p}$ consists of $r_{p}$ number of nodes and satisfies $N=$ $1+r_{2}+r_{3}, p=1,2, \ldots, m$. The Laplacian matrix of the graph $\mathcal{G}_{p}$ is recorded as $\mathcal{L}_{p}$. Including a major leader and minor leaders, an augmented topology diagram $\overline{\mathcal{G}}_{2}$ which consists of diagram $\mathcal{G}_{2}$, node 0 , and the directed edges of some nodes to node 0 is considered. An augmented topology diagram $\overline{\mathcal{G}}_{p}$ is composed of diagram $\mathcal{G}_{p}$, nodes from minor leader layer, and the directed edges of some node-to-node from the minor leader layer. Define set $v 1=\left\{1,2, \ldots, r_{2}\right\}, v 2=\left\{r_{2}+1, r_{2}+2, \ldots, r_{2}+r_{3}\right\}$.

The linear dynamics of major leader without input can be described as

$$
\dot{x}_{0}(t)=A x_{0}(t)
$$

the dynamics of minor leaders and followers are given as

$$
\dot{x}_{i}(t)=A x_{i}(t)+B u_{i}(t), i \in v 1 \cup v 2,
$$

where $x_{0}(t) \in \mathbb{R}^{n}, x_{i}(t) \in \mathbb{R}^{n}$ are the states of major leader, minor leaders and followers, respectively, $A \in \mathbb{R}^{n \times n}$ and $B \in \mathbb{R}^{n \times m}$ are constant matrices, and $u(t) \in \mathbb{R}^{m}$ is the control input.

Firstly, some basic assumptions and lemmas are given as follows:

Assumption 1. Node 0 is globally accessible in a graph $\overline{\mathcal{G}}_{2}$ that contains a major leader and $r_{2}$ minor leaders. The node from the minor leader layer is globally accessible in a graph $\overline{\mathcal{G}}_{p}$ that contains a minor leader and some followers.

Assumption 2. The matrix pair $(A, B)$ is stabilizable.

Assumption 3. Each row sum of $l_{i j}$ in $\mathcal{L}_{l}$ is zero; each row sum of $l_{i j}$ in $\mathcal{L}_{p}$ is zero.

Lemma 1. The Laplacian matrix has a simple zero eigenvalue and all the other eigenvalues have positive real parts if and only if graph $\mathcal{G}$ contains a directed spanning tree.

Definition 1. The consensus of the HLFN is considered achieved if

$$
\lim _{t \rightarrow \infty}\left\|x_{i}(t)-x_{0}(t)\right\|=0, i \in v 1 \cup v 2
$$

Remark 1. These assumptions are necessary for MAS with three-layer networks. If there is no Assumption 1, some minor leaders may be isolated from the major leader and unable to receive information from the major leader, thus detaching from the entire MAS, and several isolated smaller groups or individual isolated followers may be formed in the same follower subgroup, so that some followers cannot receive control information from the corresponding minor leaders. This obviously fails to achieve the desired control objectives.

\section{Main Results}

\subsection{Event-Based Control for Consensus of HLFNs}

In this section, the consensus of uncoupled followers under the influence of dynamic major leader is analyzed. 
For the minor leaders, the control goal is to enable the states of the minor leaders follow the major leader states. In order to achieve the consensus, the event-triggered control law for agent $i(i \in v 1)$ is designed:

$$
u_{i_{1}}(t)=K\left[\sum_{j \in \mathcal{N}_{i}} a_{i j}\left(\hat{x}_{j}(t)-\hat{x}_{i}(t)\right)+d_{i}\left(x_{0}(t)-\hat{x}_{i}(t)\right)\right], i \in v 1,
$$

where $K \in \mathbb{R}^{m \times n}$ is a feedback matrix, which is described in a later section. For the follower $i(i \in v 2)$ in the follower group, the corresponding control protocol is designed as

$$
u_{i_{2}}(t)=K\left[\sum_{j \in \mathcal{N}_{i}} a_{i j}\left(\hat{x}_{j}(t)-\hat{x}_{i}(t)\right)+d_{i}\left(\hat{x}_{p}(t)-\hat{x}_{i}(t)\right)\right], i \in v 2 .
$$

where $x_{p}(t)$ is the state of the minor leader to node $i$. Here, $\hat{x}_{i}(t)$ is defined as $x_{i}\left(t_{k}^{i}\right), t \in\left[t_{k^{\prime}}^{i} t_{k+1}^{i}\right)$.

In order to analyze the consensus problem, we define:

$$
\begin{aligned}
& \delta_{i}(t)=x_{i}(t)-x_{0}(t), i \in v 1 \cup v 2, \\
& \varepsilon_{i}(t)=\hat{x}_{i}(t)-x_{i}(t), i \in v 1 \cup v 2 .
\end{aligned}
$$

Then the system of Equation (2) can be expressed as:

$$
\begin{aligned}
\dot{\delta}_{i 1}(t) & =\dot{x}_{i}(t)-\dot{x}_{0}(t) \\
& =A \delta_{i}(t)+B K\left[\sum_{j \in \mathcal{N}_{i}} a_{i j}\left(\varepsilon_{j}(t)+\delta_{j}(t)-\varepsilon_{i}(t)-\delta_{i}(t)\right)\right. \\
& \left.-d_{i}\left(\varepsilon_{i}(t)+\delta_{i}(t)\right)\right], i \in v 1, \\
\dot{\delta}_{i 2}(t) & =\dot{x}_{i}(t)-\dot{x}_{0}(t) \\
& =A \delta_{i}(t)+B K\left[\sum_{j \in \mathcal{N}_{i}} a_{i j}\left(\delta_{j}(t)+\varepsilon_{j}(t)-\delta_{i}(t)-\varepsilon_{i}(t)\right)\right. \\
& \left.+d_{i}\left(\varepsilon_{p}(t)+\delta_{p}(t)-\delta_{i}(t)-\varepsilon_{i}(t)\right)\right], i \in v 2 .
\end{aligned}
$$

where $\dot{\delta}_{i 1}(t)$ is the error of minor leaders, and $\dot{\delta}_{i 2}(t)$ is the error of followers. Let $\delta(t)=$ $\left(\delta_{1}^{T}(t), \delta_{2}^{T}(t), \cdots, \delta_{r}^{T}(t)\right)^{T}, \varepsilon(t)=\left(\varepsilon_{1}^{T}(t), \varepsilon_{2}^{T}(t), \cdots, \varepsilon_{r}^{T}(t)\right)^{T}$, the system of Equations (8) and (9) becomes

$$
\begin{gathered}
\dot{\delta}_{1}(t)=\left[\left(I_{r_{2}} \otimes A\right)-\left(\mathcal{L}_{2}+\mathcal{D}_{2}\right) \otimes B K\right] \delta_{1}(t) \\
-\left[\left(\mathcal{L}_{2}+\mathcal{D}_{2}\right) \otimes B K\right] \varepsilon(t), i \in v 1 \\
\dot{\delta}_{2}(t)=\left[\left(I_{r_{p}} \otimes A\right)-\left(\mathcal{L}_{p}+\mathcal{D}_{p}\right) \otimes B K\right] \delta(t)-\left[\left(\mathcal{L}_{p}\right.\right. \\
\left.\left.+\mathcal{D}_{p}\right) \otimes B K\right] \varepsilon(t)+\left(\mathcal{D}_{p} \otimes B K\right)\left(\delta_{p}(t)+\varepsilon_{p}(t)\right), i \in v 2 .
\end{gathered}
$$

Theorem 1. Suppose that Assumptions 1, 2 and 3 hold. Consider the HLFN Equations (1) and (2) with the control in Equations (4) and (5). The consensus problem can be solved if:

$$
\begin{gathered}
f_{i_{1}}(t)=\left\|\varepsilon_{i}(t)\right\|-\left\|B K q_{i}(t)\right\|-\beta e^{-\gamma\left(t-t_{0}\right)}, i \in v 1 . \\
f_{i_{2}}(t)=\left\|\varepsilon_{i}(t)\right\|-\beta e^{-\gamma\left(t-t_{0}\right)} \\
-\frac{\left\|B K q_{i}(t)\right\|-2\left\|B K \sum_{j \in \mathcal{N}_{i}} d_{i}\left(\hat{x}_{p}(t)-x_{0}(t)\right)\right\|}{2\left\|\left(L_{P}+D\right) \otimes B K\right\|}, i \in v 2 .
\end{gathered}
$$


and

$$
\begin{aligned}
& q_{i}(t)=\sum_{j \in \mathcal{N}_{i}} a_{i j}\left(\hat{x}_{j}(t)-\hat{x}_{i}(t)\right)+d_{i}\left(x_{0}(t)-\hat{x}_{i}(t)\right), i \in v 1, \\
& q_{i}(t)=\sum_{j \in \mathcal{N}_{i}} a_{i j}\left(\hat{x}_{j}(t)-\hat{x}_{i}(t)\right)+d_{i}\left(\hat{x}_{p}(t)-\hat{x}_{i}(t)\right), i \in v 2 .
\end{aligned}
$$

For any $\beta>0$, and $K=B^{T} P, P$ is a positive-definitive matrix.

Proof of Theorem 1. Using the variation of parameter formula, Equation (10) becomes

$$
\begin{aligned}
\delta(t) & =e^{\left[\left(I_{r} \otimes A\right)-\left(\mathcal{L}_{2}+\mathcal{D}_{2}\right) \otimes B K\right]\left(t-t_{0}\right)} \delta\left(t_{0}\right) \\
& +\int_{t_{0}}^{t} e^{\left[\left(I_{r} \otimes A\right)-\left(\mathcal{L}_{2}+\mathcal{D}_{2}\right) \otimes B K\right](t-\theta)} \\
& \times\left(-\left(\left(\mathcal{L}_{2}+\mathcal{D}_{2}\right) \otimes B K\right)\right) \varepsilon(\theta) d \theta .
\end{aligned}
$$

In reality, if $(A, B)$ is stabilizable and the communication topology of these agents in the leader-follower structure has a directed spanning tree, it can be obtained from Lemma 1 that all the eigenvalues of $I_{r} \otimes A-\left(\mathcal{L}_{2}+\mathcal{D}_{2}\right) \otimes B K$ have non-positive real parts. Therefore, positive constants $a$ and $\rho$ for $t \geq t_{0}$ are existed, such that (refer to [35])

$$
\left\|e^{\left[I_{r_{2}} \otimes A-\left(\mathcal{L}_{2}+\mathcal{D}_{2}\right) \otimes B K\right]\left(t-t_{0}\right)}\right\| \leq a e^{-\rho\left(t-t_{0}\right)} .
$$

Furthermore, we can get

$$
\begin{aligned}
\|\delta(t)\| & \leq \alpha_{1} e^{-\gamma_{1}\left(t-t_{0}\right)}\left\|\delta\left(t_{0}\right)\right\| \\
& +\alpha_{1} \int_{t_{0}}^{t} e^{-\gamma_{1}(t-\theta)}\left\|\left(\mathcal{L}_{2}+\mathcal{D}_{2}\right) \otimes B K\right\|\|\tilde{x}(\theta)\| d \theta,
\end{aligned}
$$

Forced by Equation (12), we can get

$$
\|\varepsilon(t)\| \leq \tilde{\alpha}\|\delta(t)\|+\tilde{\beta} e^{-\gamma\left(t-t_{0}\right)},
$$

Combine Equation (14) with Equation (15), it holds

$$
\begin{aligned}
\|\delta(t)\| & \leq \alpha_{1}\left\|\delta\left(t_{0}\right)\right\| e^{-\rho\left(t-t_{0}\right)}+\alpha_{1} \int_{t_{0}}^{t} e^{-\rho(t-\theta)} \\
& \times\left\|\left(\mathcal{L}_{2}+\mathcal{D}_{2}\right) \otimes B K\right\|\left(\tilde{\alpha}\|\delta(\theta)\|+\tilde{\beta} e^{-\gamma\left(\theta-t_{0}\right)}\right) d \theta .
\end{aligned}
$$

Then, we prove that

$$
\|\delta(t)\|<\eta \alpha_{1}\left\|\delta\left(t_{0}\right)\right\| e^{-\gamma\left(t-t_{0}\right)}
$$

If Equation (17) does not hold, then $t^{*}>t_{0}$ for $\left\|\delta\left(t^{*}\right)\right\|=v\left(t^{*}\right)$ and $\|\delta(t)\|<v(t)$ for $t<t^{*}$. Then by Equation (16), one has 


$$
\begin{aligned}
v\left(t^{*}\right) & =\left\|\delta\left(t^{*}\right)\right\| \\
& <\eta \alpha_{1} e^{-\rho\left(t^{*}-t_{0}\right)}\left\|\delta\left(t_{0}\right)\right\|+\eta \alpha_{1} \int_{t_{0}}^{t^{*}} e^{-\rho\left(t^{*}-\theta\right)} \\
& \times\left(\tilde{\alpha}\|\delta(\theta)\|+\tilde{\beta} e^{-\gamma\left(\theta-t_{0}\right)}\right) d \theta \\
& <\eta \alpha_{1} e^{-\rho\left(t^{*}-t_{0}\right)}\left\|\delta\left(t_{0}\right)\right\|+\eta \alpha_{1}\left(\tilde{\alpha} \alpha_{1}\left\|\delta\left(t_{0}\right)\right\|+\tilde{\beta}\right) \\
& \times\left(e^{-\gamma\left(t^{*}-t_{0}\right)}-e^{-\rho\left(t^{*}-t_{0}\right)}\right) \\
& <\eta \alpha_{1}\left\|\delta\left(t_{0}\right)\right\| e^{-\gamma\left(t^{*}-t_{0}\right)} \\
& =v\left(t^{*}\right) .
\end{aligned}
$$

The conflict of Equation (18) demonstrates that Equation (17) is well-founded, which implies that the consensus of the system in Equation (1), Equation (2) can be achieved exponentially.

Then using the variation of parameter formula, Equation (11) can be rewritten as

$$
\begin{aligned}
\varepsilon(t) & =e^{\left[\left(I_{r_{p}} \otimes A\right)-\left(\mathcal{L}_{p}+\mathcal{D}_{p}\right) \otimes B K\right]\left(t-t_{0}\right)} \varepsilon\left(t_{0}\right) \\
& +\int_{t_{0}}^{t} e^{\left[\left(I_{r_{p}} \otimes A\right)-\left(\mathcal{L}_{p}+\mathcal{D}_{p}\right) \otimes B K\right](t-\theta)} \\
& \times\left[\left(-\left(\left(\mathcal{L}_{p}+\mathcal{D}_{p}\right) \otimes B K\right)\right) e(\theta)\right. \\
& \left.+\left(\mathcal{D}_{p} \otimes B K\right)(\tilde{x}(\theta)+\delta(\theta))\right] d \theta .
\end{aligned}
$$

It follows from Equation (19)

$$
\begin{aligned}
\|\varepsilon(t)\| & \leq \alpha_{2} e^{-\rho\left(t-t_{0}\right)}\left\|\varepsilon\left(t_{0}\right)\right\| \\
& +\alpha_{2} \int_{t_{0}}^{t} e^{-\rho(t-\theta)}\left[\left\|\left(\mathcal{L}_{p}+\mathcal{D}_{p}\right) \otimes B K\right\|\|\varepsilon(\theta)\|\right. \\
& \left.+\left\|\mathcal{D}_{p} \otimes B K\right\|\|\varepsilon(\theta)+\delta(\theta)\|\right] d \theta .
\end{aligned}
$$

Replacing Equation (15) in Equation (20),

$$
\begin{aligned}
\|\varepsilon(t)\| & \leq \alpha_{2} e^{-\rho\left(t-t_{0}\right)}\left\|\varepsilon\left(t_{0}\right)\right\| \\
& +\alpha_{2} \int_{t_{0}}^{t} e^{-\rho(t-\theta)}\left[\left\|\left(\mathcal{L}_{p}+\mathcal{D}_{p}\right) \otimes B K\right\|\|\varepsilon(\theta)\|\right. \\
& \left.+r \beta e^{-\gamma\left(\theta-t_{0}\right)}\right] d \theta .
\end{aligned}
$$

Then, we prove that

$$
\|\varepsilon(t)\|<\eta \alpha_{2}\left\|\varepsilon\left(t_{0}\right)\right\| e^{-\gamma\left(t-t_{0}\right)} .
$$

This part of the proof is equivalent to the previous process, which is omitted here.

To avoid zeno-behavior, we need to get the lower boundary of the positive constant for the interval between any two events $\Delta t_{i}$, suppose agent $i$ triggered at the moment $t_{i}^{*} \geq 0$, then $e_{i}\left(t_{i}^{*}\right)=0$. For between the time $t_{i}^{*}$ and the time $t_{i}$ which the next event is triggered, we have

$$
\dot{e}_{i}(t)=-\dot{x}_{i}(t)=-\left[A x_{i}(t)+B u_{i}(t)\right] .
$$

By integrating the two sides of the above equation, we can get

$$
\left|e_{i}(t)\right|=\int_{t^{*}}^{t}\left|A x_{i}(s)+B u_{i}(s)\right| d s .
$$


we know

$$
\begin{aligned}
\left|A x_{i}(t)+B u_{i}(t)\right| & \leq\left\|\left(I_{r} \otimes A\right)\right\|\|x(t)\| \\
& +\left\|\left(\mathcal{L}_{p}+\mathcal{D}_{p}\right) \otimes B K\right\|(\|\varepsilon(t)\|+\|e(t)\| \\
& +\left\|\mathcal{D}_{p} \otimes B K\right\|\|\delta(t)\| \\
& =c .
\end{aligned}
$$

where $c$ is a normal value.

By Equations (24) and (25), $\left|e_{i}(t)\right| \leq c\left(t-t^{*}\right)$ can be obtained. Assume $c\left(t-t^{*}\right)=c_{1}$, when the error vector decreases from $\left|e_{i}(t)\right|=c_{1}$ to $\left|e_{i}(t)\right|=0$, the next event trigger will occur. Therefore, there exists a strict positive lower bound $\Delta t_{i}=t_{i}-t_{i}^{*}=c_{1} / c$ between the triggering times of two events. Since there exists such a lower bound for arbitrary agent and any event time, we believe that zeno-behavior will not exist. The process of proof for Theorem 1 is thus completed.

\subsection{Event-Based Control for Consensus of HLFNs with Layer-to-Layer Delays}

In this part, we consider the result in case when the layer-to-layer delays exist. For the minor leaders, the following protocol for agent $i$ is designed:

$$
\begin{array}{r}
u_{i}(t)=K\left[\sum_{j \in \mathcal{N}_{i}} a_{i j}\left(\hat{x}_{j}(t)-\hat{x}_{i}(t)\right)+d_{i}\left(x_{0}\left(t-\tau_{1}(t)\right)-\hat{x}_{i}(t)\right)\right], \\
i \in v 1, \\
\left.u_{i}(t)=\underset{j \in \mathcal{N}_{i}}{K[} a_{i j}\left(\hat{x}_{j}(t)-\hat{x}_{i}(t)\right)+d_{i}\left(\hat{x}_{p}\left(t-\tau_{2}(t)\right)-\hat{x}_{i}(t)\right)\right], \\
i \in v 2 .
\end{array}
$$

In this part, we consider layer-to-layer delays in communication. Define that the communication between major leader and minor leaders is subject to a time delay $\tau_{1}(t)$, which satisfies $0<\tau_{1}(t)<\mu_{1}$, the communication between minor leader and followers is subject to a time delay $\tau_{2}(t)$, which satisfies $0<\tau_{2}(t)<\mu_{2}$.

Theorem 2. Suppose that Assumptions 1, 2 and 3 hold. Consider the HLFN Equations (1) and (2) with control, (26) and (27), the consensus problem can be solved if:

$$
\begin{aligned}
f_{i}(t) & =\left\|\varepsilon_{i}(t)\right\|-\beta e^{-\gamma\left(t-t_{0}\right)} \\
& -\frac{\left\|B K q_{i}(t)\right\|-2\left\|B K \sum_{q \in \mathcal{N}_{p}} d_{i}\left(\hat{x}_{p}(t)-x_{0}\left(t-\tau_{1}(t)\right)\right)\right\|}{2\left\|\left(\mathcal{L}_{2}+\mathcal{D}_{2}\right) \otimes B K\right\|}, \\
i \in v 1, & \\
f_{i}(t)= & \left\|\varepsilon_{i}(t)\right\|-\beta e^{-\gamma\left(t-t_{0}\right)} \\
& -\frac{\left\|B K q_{i}(t)\right\|-2\left\|B K \sum_{j \in \mathcal{N}_{i}} d_{i}\left(\hat{x}_{p}(t)-x_{0}\left(t-\tau_{2}(t)\right)\right)\right\|}{2\left\|\left(\mathcal{L}_{p}+\mathcal{D}_{p}\right) \otimes B K\right\|}, \\
i \in v 2 . &
\end{aligned}
$$

For any $\beta>0$, and $K=B^{T} P, P$ is a positive-definitive matrix. 
Proof of Theorem 2. In this part, we divide the proof process into two parts, for minor leader $i\left(i=1,2, \ldots r_{2}\right)$, we can get

$$
\begin{aligned}
\|\delta(t)\| & \leq \alpha_{1} e^{-\rho\left(t-t_{0}\right)}\left\|\delta\left(t_{0}\right)\right\| \\
& +\alpha_{1} \int_{t_{0}}^{t} e^{-\rho(t-\theta)}\left\|\left(\mathcal{L}_{2}+\mathcal{D}_{2}\right) \otimes B K\right\|\left\|\varepsilon_{i}(\theta)\right\| \\
& \left.-\left\|\left(\mathcal{D}_{2} \otimes B K\right)\right\|\left\|\left(e^{A \theta}-e^{A\left(\theta-\tau_{1}(\theta)\right)}\right)\right\|\right) d \theta
\end{aligned}
$$

forced by Equation (28), one can get

$$
\|\delta(t)\|<\eta \alpha_{1} e^{-\gamma\left(t-t_{0}\right)}\left\|\delta\left(t_{0}\right)\right\|,
$$

For follower $i(i \in v 2)$, it holds

$$
\begin{aligned}
\|\delta(t)\| & \leq \alpha_{2} e^{-\rho\left(t-t_{0}\right)}\left\|\delta\left(t_{0}\right)\right\|+\alpha_{2} \int_{t_{0}}^{t} e^{-\rho(t-\theta)} \\
& \times\left[\left\|\left(\mathcal{L}_{p}+\mathcal{D}_{p}\right) \otimes B K\right\|\|\varepsilon(\theta)\|+\left\|\mathcal{D}_{2} \otimes B K\right\|\right. \\
& \left.\times\left\|\delta\left(\theta-\tau_{2}(\theta)\right)+\varepsilon_{p}\left(\theta-\tau_{2}(\theta)\right)-\left(e^{A \theta}-e^{A\left(\theta-\tau_{2}(\theta)\right)}\right)\right\|\right] d \theta,
\end{aligned}
$$

It can be concluded from Equation (29) that

$$
\|\delta(t)\|<\eta \alpha_{2}\left\|\delta\left(t_{0}\right)\right\| e^{-\gamma\left(t-t_{0}\right)} .
$$

The proof of zeno-behavior is equivalent to that of Theorem 1, which is omitted here. The proof of Theorem 2 is thus completed.

Remark 2. The conclusion of this paper is that, based on the three-layer hierarchical structure, agents from the minor leader layer and the follower layer only interacts with the same layer agents and direct leaders, therefore, the analysis of three-layer network can be extended to multi-layer hierarchical MAS.

\section{Simulation Results}

In this section, two numerical example are shown to demonstrate the corresponding analysis of the results gained in the previous section. Consider the event-based consensus of HLFNs with thirteen agents, a major leader, three minor leaders, and nine followers. The communication topology is described as seen in Figure 1 and the connectivity weights are given as $a_{i j}=1, d_{i j}=1$, the other weights are all equal to zero. From the topology graph and the matrix relationship given, it can be seen that node 0 and nodes from the second layer are globally reachable in $\overline{\mathcal{G}}_{2}$ and $\overline{\mathcal{G}}_{p}$, respectively. Moreover, there is a directed spanning tree in the interconnection graph. Assume that

$$
\begin{gathered}
A=\left[\begin{array}{ccc}
-6 & 3 & 3 \\
3 & -3 & 0 \\
-3 & 0 & 0
\end{array}\right], B=\left[\begin{array}{c}
0 \\
0.2 \\
0
\end{array}\right] \\
P=\left[\begin{array}{lll}
0.3492 & 0.4488 & 0.2378 \\
0.4488 & 0.9798 & 0.4697 \\
0.2378 & 0.4697 & 0.4827
\end{array}\right]
\end{gathered}
$$

Thus $(\mathrm{A}, \mathrm{B})$ is stabilizable. Consider the hierarchical leader-follower networks with the interconnection graph given in Figure 1, for any initial condition, Figures $2-4$ show the change of error of each agents. It can be seen from Figures 2-4 that the consensus can be achieved eventually under the proposed event-triggered strategy. The enlarged part of the figure shows that the system 
reached consensus in about $11 \mathrm{~s}$. Event time instants are shown in Figure 5, in which no Zeno triggering behaviors or even dense events for any agent are observed. Figures 6-8 shows the the change of error of agents with layer-to-layer delays, which shows the consensus can be achieved eventually under the proposed event-triggered strategy with layer-to-layer delays in about $15 \mathrm{~s}$. Due to the interference of the layer-to-layer delays, the time to reach the consensus in Figures 6-8 is longer than that in Figures 2 and 3. Figure 9 shows the event time instants under the event-triggered law with layer-to-layer delays. As can be seen from Figures 5 and 9, leader 1-3 triggers less than other followers, which greatly saves the waste of system resources. From the simulation results, one can see that Theorem 2 does more controller updates than that in Theorem 1.

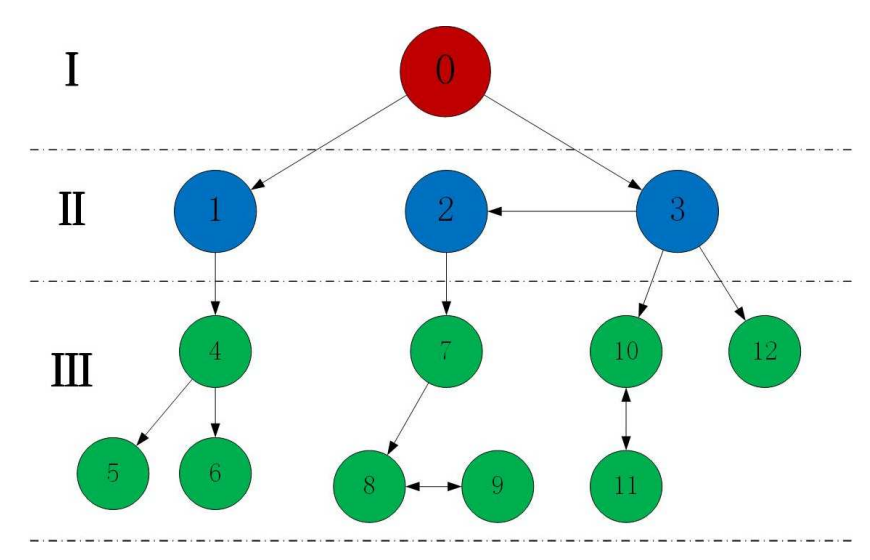

Figure 1. Topology of the hierarchical multi-agent network with uncoupled follower groups.

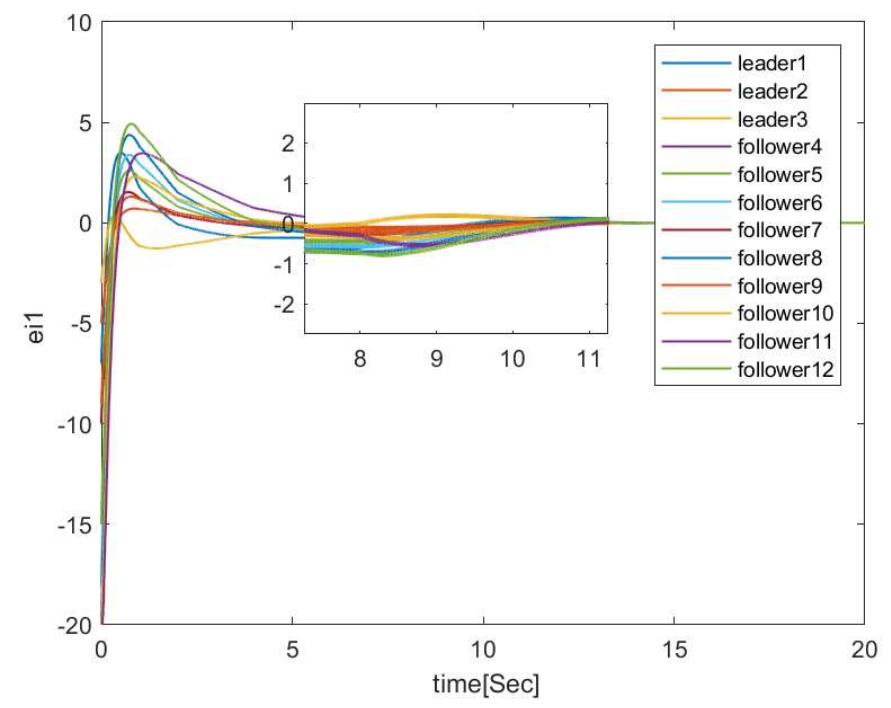

Figure 2. Position State Component 1 of Agent without delay. 


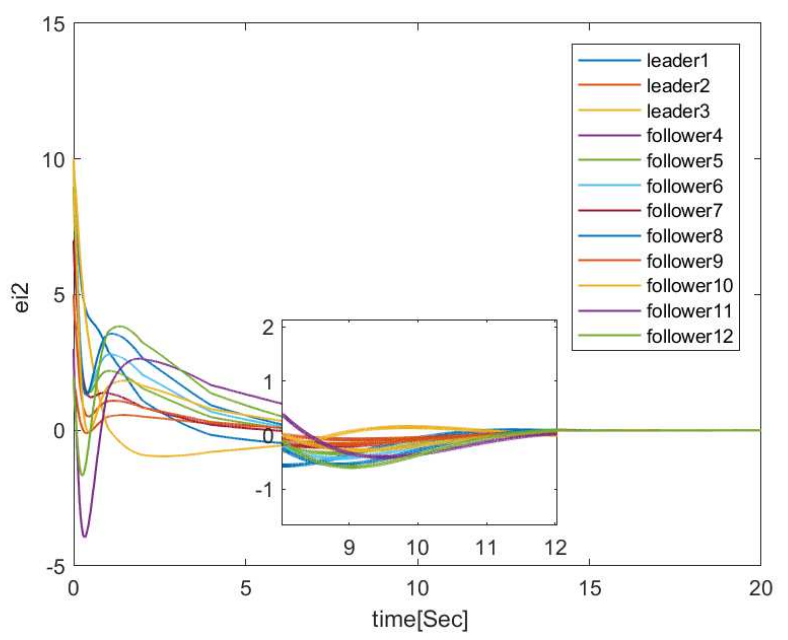

Figure 3. Position State Component 2 of Agent without delay.

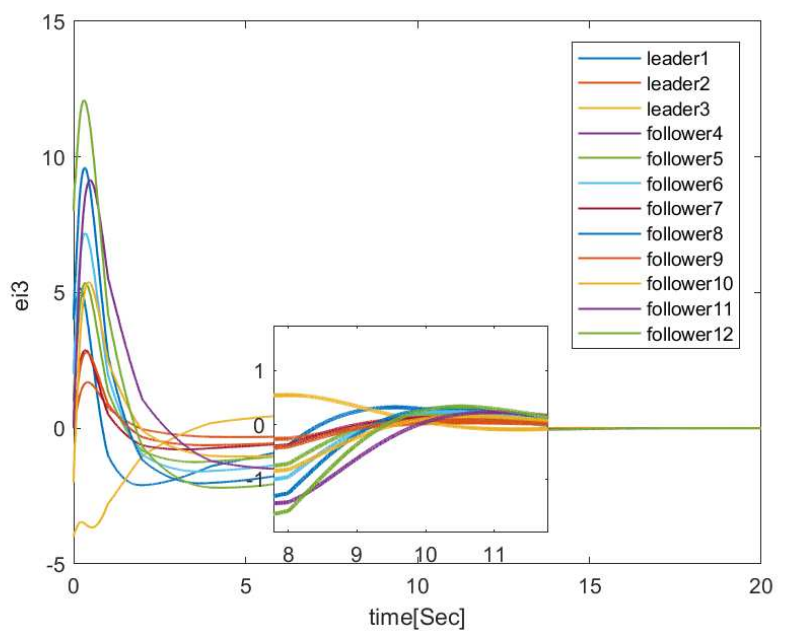

Figure 4. Position State Component 3 of Agent without delay.

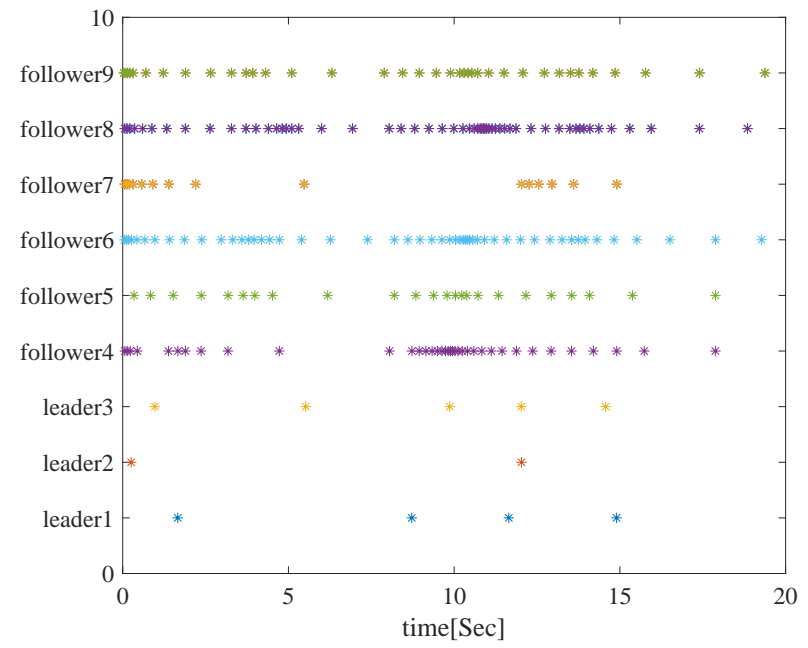

Figure 5. Event-trigger numbers under the event-based control law of Equation (4). 


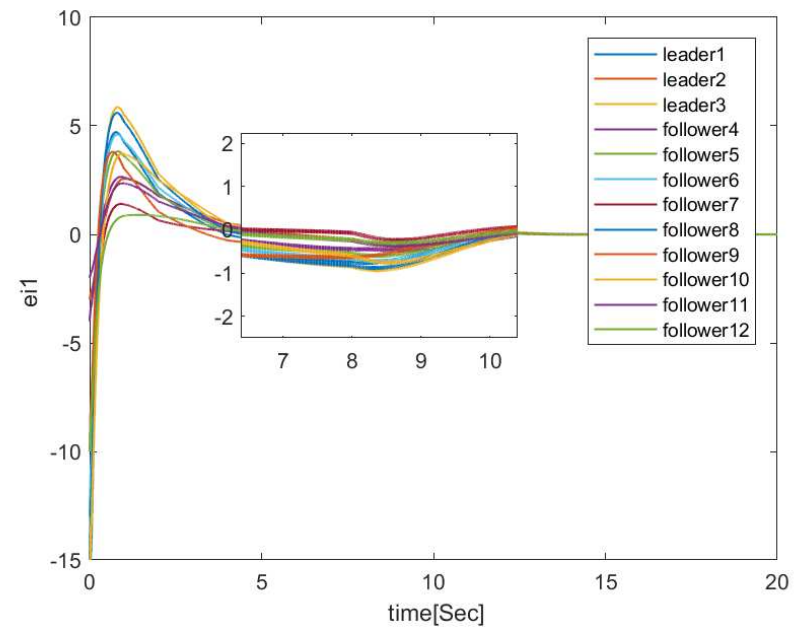

Figure 6. Position State Component 1 of Agent with hierarchical delay.

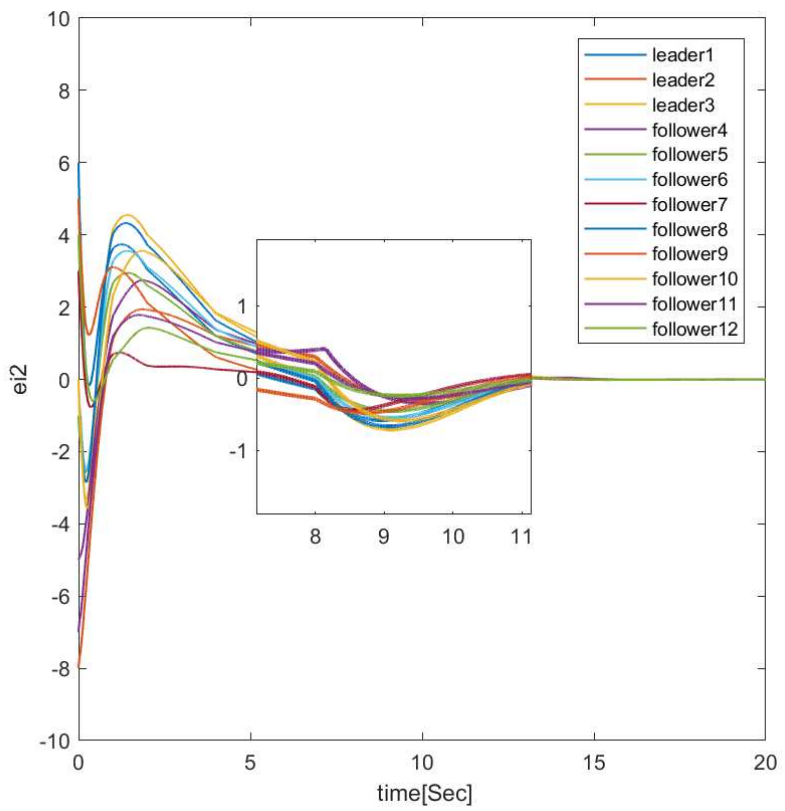

Figure 7. Position State Component 2 of Agent with hierarchical delay. 


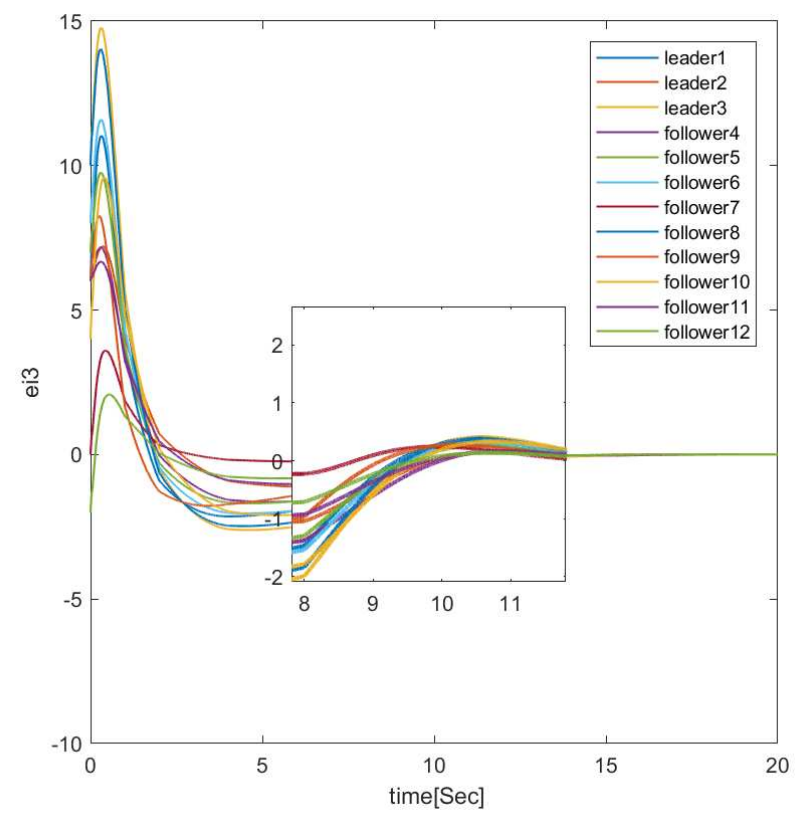

Figure 8. Position State Component 3 of Agent with hierarchical delay.

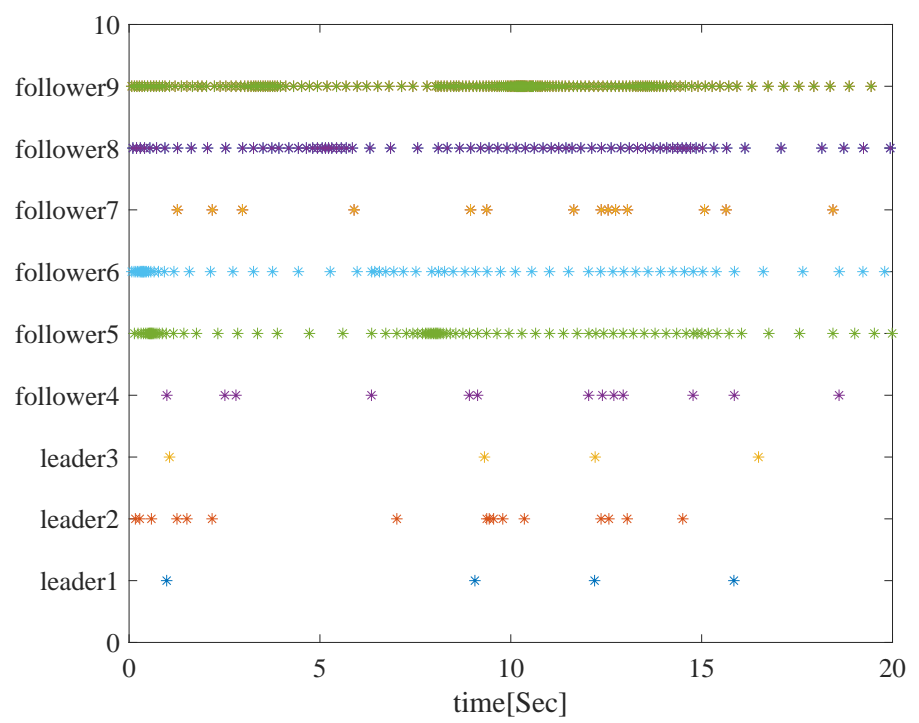

Figure 9. Event-trigger numbers under the event-based control law of Equation (5) with layer-to-layer delay.

Remark 3. The state of the agents in this paper is three-dimensional, and Figures 2-4 describe the state changes of the three dimensions, respectively.

\section{Conclusions}

In this paper, a novel hierarchical event-based control for hierarchical leader-follower networks is proposed. Based on matrix theory, algebraic graph theory, Lyapunov theory, and under the given event-triggered condition, it is proven that the protocols can realize consensus. Moreover, we show that the system can reach consensus with the layer-to-layer delays. Finally, simulation results are presented to support the theorems. In the future, more effort will be made towards relaxing the connectivity 
condition on the topology of the multi-agent network. The restrictions on the time delays may be lifted. How to extend consensus to multi-consensus is another topic in the future.

Author Contributions: Conceptualization and methodology, G.-H.X., M.X., and M.-F.G.; software, F.Q.; validation, M.L.; writing-review and editing, T.-F.D. Finally, All authors have read and agreed to the published version of the manuscript.

Funding: This work was supported by National Natural Science Foundation of China(61603127, 61703374).

Conflicts of Interest: The authors declare no conflict of interest.

\section{References}

1. Zheng, Y.; Ma, J.; Wang, L. Consensus of hybrid multi-agent systems. IEEE Trans. Neural Netw. Learn. Syst. 2017, 29, 1359-1365. [CrossRef] [PubMed]

2. Liu, X.; Zhang, K.; Xie, W.C. Consensus of multi-agent systems via hybrid impulsive protocols with time-delay. Nonlinear Anal. Hybrid Syst. 2018, 30, 134-146. [CrossRef]

3. Yan, Z.; Pan, X.; Yang, Z.; Yue, L. Formation Control of Leader-Following Multi-UUVs With Uncertain Factors and Time-Varying Delays. IEEE Access 2019, 7, 118792-118805. [CrossRef]

4. Ding, Y.; Wang, X.; Cong, Y.; Li, H. Scalability Analysis of Algebraic Graph-Based Multi-UAVs Formation Control. IEEE Access 2019, 7, 129719-129733. [CrossRef]

5. Tanner, H.G.; Christodoulakis, D.K. Decentralized cooperative control of heterogeneous vehicle groups. Robot. Auton. Syst. 2007, 55, 811-823. [CrossRef]

6. Liang, C.D.; Wang, L.; Yao, X.Y.; Liu, Z.W. Multi-target tracking of networked heterogeneous collaborative robots in task space. Nonlinear Dynam. 2019, 1-15. [CrossRef]

7. HYazdani, S.; Haeri, M. Flocking of multi-agent systems with multiple second-order uncoupled linear dynamics and virtual leadere. IET Control Theory Appl. 2016, 10, 853-860. [CrossRef]

8. Ren, W.; Beard, R.W.; Atkins, E.M. Information consensus in multivehicle cooperative control. IEEE Control Syst. Mag. 2007, 27, 71-82.

9. Olfati-Saber, R.; Fax, J.A.; Murray, R.M. Consensus and cooperation in networked multi-agent systems. Proc. IEEE 2007, 95, 215-233. [CrossRef]

10. Wang, X.; Su, H. Self-triggered leader-following consensus of multi-agent systems with input time delay. Neurocomputing 2019, 330, 70-77. [CrossRef]

11. Shen, Q.; Shi, P.; Zhu, J.; Zhang, L. Adaptive consensus control of leader-following systems with transmission nonlinearities. Int. J. Control 2019, 92, 317-328. [CrossRef]

12. Ning, B.; Han, Q.L.; Lu, Q. Fixed-Time Leader-Following Consensus for Multiple Wheeled Mobile Robots. IEEE Trans. Cybern. 2019. [CrossRef] [PubMed]

13. Nagy, M.; Akos, Z.; Biro, D.; Vicsek, T. Hierarchical group dynamics in pigeon flocks. Nature 2010, 464, 890. [CrossRef] [PubMed]

14. He, D.X.; Xu, G.H.; Guan, Z.H.; Chi, M.I. Hybrid coordination of multi-agent networks with hierarchical leaders. Commun. Nonlinear Sci. Numer. Simul. 2015, 27,110-119. [CrossRef]

15. Rakkiyappan, R.; Chandrasekar, A.; Laksmanan, S.; Park, J.H. State estimation of memristor-based recurrent neural networks with time-varying delays based on passivity theory. Complexity 2014, 19, 32-43. [CrossRef]

16. Li, Y.; Li, H.; Ding, X.; Zhao, G. Leader-Follower Consensus of Multiagent Systems With Time Delays Over Finite Fields. IEEE Trans. Cybern. 2018, 49, 3203-3208. [CrossRef]

17. Zhao, G.; Hua, C.; Guan, X. Distributed Event-Triggered Consensus of Multiagent Systems With Communication Delays: A Hybrid System Approach. IEEE Trans. Cybern. 2019. [CrossRef]

18. Jiang, Y.; Zhang, Y.; Wang, S. Distributed adaptive consensus control for networked robotic manipulators with time-varying delays under directed switching topologies. Peer-to-Peer Netw. Appl. 2019. [CrossRef]

19. Yu, H.; Chen, X.; Chen, T.; Hao, F. Event-triggered bipartite consensus for multi-agent systems: A Zeno-free analysis. IEEE Trans. Autom. Control 2019. [CrossRef]

20. Yi, X.; Yang, T.; Wu, J.; Johansson, K.H. Distributed event-triggered control for global consensus of multi-agent systems with input saturation. Automatica 2019, 310, 1-9. [CrossRef]

21. Ren, J.; Song, Q.; Lu, G. Event-triggered bipartite leader-following consensus of second-order nonlinear multi-agent systems under signed digraph. IEEE J. Frankl. Inst. 2019, 356, 6591-6609. [CrossRef] 
22. Mirollo, R.E.; Strogatz, S.H. Synchronization of pulse-coupled biological oscillators. SIAM J. Appl. Math. 1990, 50, 1645-1662. [CrossRef]

23. Strogatz, S.H. From Kuramoto to Crawford: Exploring the onset of synchronization in populations of coupled oscillators. Phys. D Nonlinear Phenom. 2000, 143, 1-20. [CrossRef]

24. Preciado, V.M.; Verghese, G.C. Synchronization in Generalized Erd ö sR é nyi Networks of Nonlinear Oscillators. In Proceedings of the 44th IEEE Conference on Decision and Control, Seville, Spain, 12-15 December 2005; pp. 4628-4633.

25. Sepulchre, R.; Paley, D.; Leonard, N. Collective motion and oscillator synchronization. Lect. Notes Control Inf. Sci. 2004, 309, 466-469.

26. Rojo-Rodriguez, E.G.; Garcia, O.; Ollervides, E.J.; Zambrano Robledo, P.C. Robust Consensus-Based Formation Flight for Multiple Quadrotors. J. Intell. Robot. Syst. 2019, 93, 213-226. [CrossRef]

27. Masroor, S.; Peng, C. Event triggered non-inverting chopper fed networked DC motor speed synchronization. COMPEL Int. J. Comput. Math. Electr. Electron. Eng. 2018, 37, 911-929. [CrossRef]

28. Zhang, H.; Yang, R.; Yan, H. Distributed event-triggered control for consensus of multi-agent systems. J. Frankl. Inst. 2015, 352, 3476-3488. [CrossRef]

29. Wang, Y.W.; Lei, Y.; Bian, T.; Guan, Z.H. Distributed Control of Nonlinear Multiagent Systems With Unknown and Nonidentical Control Directions via Event-Triggered Communication. IEEE Trans. Cybern. 2019. [CrossRef]

30. Jian, L.; Hu, J.; Wang, J. New Event-based Control for Sampled-data Consensus of Multi-agent Systems. Int. J. Control Autom. Syst. 2019, 17, 1107-1116. [CrossRef]

31. Lai, Q.; Didier Kamdem Kuate, P.; Liu, F.; Ho-Ching Iu, H. An extremely simple chaotic system with infinitely many coexisting attractors. IEEE Trans. Circuits Syst. II Express Briefs 2019. [CrossRef]

32. Lai, Q.; Akgul, A.; Li, C.; Xu, G.; Çavuşoğlu, Ü. A New Chaotic System with Multiple Attractors: Dynamic Analysis, Circuit Realization and S-Box Design. Entropy 2018, 20, 12. [CrossRef]

33. Lai, Q.; Norouzi, B.; Liu, F. Dynamic analysis, circuit realization, control design and image encryption application of an extended Lü system with coexisting attractors. Chaos Solitons Fractals 2018, 114, $230-245$. [CrossRef]

34. Ge, M.F.; Liu, Z.W.; Wen, G.; Yu, X.; Huang, T. Hierarchical Controller-Estimator for Coordination of Networked Euler-Lagrange Systems. IEEE Trans. Cybern. 2019. [CrossRef] [PubMed]

35. Zhu, W.; Sun, C.; Li, H. Event-based leader-following consensus of multi-agent systems with switching topologies. In Proceedings of the Chinese Control Conference (CCC), Hangzhou, China, 28-30 July 2015; pp. 7511-7516.

(C) 2020 by the authors. Licensee MDPI, Basel, Switzerland. This article is an open access article distributed under the terms and conditions of the Creative Commons Attribution (CC BY) license (http:/ / creativecommons.org/licenses/by/4.0/). 